\title{
On random presentations with fixed relator length
}

\author{
C. J. Ashcroft, Colva M. Roney-Dougal
}

July 29, 2019

\begin{abstract}
The standard $(n, k, d)$ model of random groups is a model where the relators are chosen randomly from the set of cyclically reduced words of length $k$ on an $n$-element generating set. Gromov's density model of random groups considers the case where $n$ is fixed, and $k$ tends to infinity. We instead fix $k$, and let $n$ tend to infinity. We prove that for all $k \geq 2$ at density $d>1 / 2$ a random group in this model is trivial or cyclic of order two, whilst for $d<\frac{1}{2}$ such a random group is infinite and hyperbolic. In addition we show that for $d<\frac{1}{k}$ such a random group is free, and that this threshold is sharp. These extend known results for the triangular $(k=3)$ and square $(k=4)$ models of random groups.
\end{abstract}

\section{Introduction and statement of results}

Gromov's density model of random groups is a famous construction in modern group theory, introduced in [3] to answer the question of what a "generic" group looks like. Models of random groups have also been used to construct exotic groups, as in [4].

Let $n \geq 2, k \geq 2$, and $0<d<1$. Randomly uniformly select a set $R$ of distinct cyclically reduced words of length $k$ over the alphabet $\left\{a_{1}, \ldots, a_{n}\right\}$ of size $N=\left\lfloor(2 n-1)^{k d}\right\rfloor$ from amongst all such sets. Then let $G=\left\langle a_{1}, \ldots, a_{n} \mid R\right\rangle$. The group $G$ is a random group in the standard $(n, k, d)$ model of random groups. If we take the set $R$ to contain only positive words over the alphabet (that is, words where no letters are inverses of the generators), we obtain the positive $(n, k, d)$ model, introduced by Odrzygóźdź. By an $(n, k, d)$ model we mean either the standard or the positive $(n, k, d)$ model.

Gromov showed (see also [8]) that for any fixed $n \geq 2$, with probability tending to 1 as $k$ tends to $\infty$, a random group in the standard $(n, k, d)$ model is trivial or isomorphic to $\mathbb{Z}_{2}$ for $d>\frac{1}{2}$, and infinite, hyperbolic, and torsion free for $d<\frac{1}{2}$, so that $d=\frac{1}{2}$ is a sharp phase transition for the density model as $k \rightarrow \infty$.

Definition 1.1. Let $k \in \mathbb{N}_{\geq 2}$, and let $0<d<1$. Let $\mathcal{P}$ be a property of groups preserved by isomorphism, and let $\mathcal{M}(n, k, d)$ be an $(n, k, d)$ model. If

$$
\lim _{n \rightarrow \infty} P(\text { A random group in } \mathcal{M}(n, k, d) \text { satisfies } \mathcal{P})=1,
$$

we will say that at density $d$, asymptotically almost surely (a.a.s.), a random group in the $\mathcal{M}(n, k, d)$ model satisfies $\mathcal{P}$. We shall also call the standard $(n, k, d)$-model as $n \rightarrow \infty$ the standard $k$-angular model at density $d$, with a comparable definition of the positive $k$-angular model.

Żuk studied the standard 3-angular model, also known as the triangular model, showing in [14] that at density $d<\frac{1}{2}$ a.a.s. a group in the triangular model is hyperbolic. He also showed that at density $d>\frac{1}{3}$ such a group satisfies Property (T) (and so is not free). It is also known (see [1]) that 
at density $d<\frac{1}{3}$ a.a.s. a group in the triangular model is isomorphic to $F_{m}$ for some $m \in \mathbb{N}$. In [6], Kotowski and Kotowski studied the positive triangular model, proving that at density $d>\frac{1}{3}$ these groups have Property (T) and so are not free.

The standard 4-angular model is also known as the the square model. This was studied more recently by Odrzygóźdź in [7], who showed at at $d>\frac{1}{2}$ a.a.s. a group in the square model is cyclic of order two, whilst at $d<\frac{1}{2}$ a.a.s. such a group is hyperbolic. Furthermore, at density $d<\frac{1}{4}$ a.a.s. a group in the square model is isomorphic to $F_{m}$ for some $m \in \mathbb{N}$. In [7], Odrzygóźdź also introduced and studied the positive square model, showing that at density $d>\frac{1}{2}$ a.a.s. a group in this model is cyclic of order four, whilst at $d<\frac{1}{2}$ a.a.s. such a group is hyperbolic. Furthermore, at $d<\frac{1}{4}$ a.a.s. a random group in the positive square model is isomorphic to $F_{m}$ for some $m \in \mathbb{N}$.

This paper generalises this work to all values of $k$. For the standard $k$-angular model we have the following:

Theorem 1. Let $k \geq 2$, and let $G$ be a random group in the standard $(n, k, d)$ model.

i) If $d>\frac{1}{2}$ then asymptotically almost surely $G$ is trivial ( $k$ odd) or cyclic of order two ( $k$ even).

ii) If $d<\frac{1}{2}$ then asymptotically almost surely $G$ is hyperbolic, infinite and torsion-free.

As a side-effect of our proof techniques, we also show the following.

Theorem 2. Let $k \geq 2$, and let $G$ be a random group in the positive $(n, k, d)$ model.

i) If $d>\frac{1}{2}$ then asymptotically almost surely $G$ is cyclic of order $k$.

ii) If $d<\frac{1}{2}$ then asymptotically almost surely $G$ is hyperbolic, infinite and torsion-free.

Furthermore, we consider freeness.

Theorem 3. Let $k \geq 2$, and let $G$ be a random group in an $(n, k, d)$ model. If $d<\frac{1}{k}$ then asymptotically almost surely $G$ is isomorphic to $F_{m}$ for some $m \in \mathbb{N}$, whilst if $d>\frac{1}{k}$ the group $G$ is asymptotically almost surely not isomorphic to a nontrivial free group.

The paper is structured as follows. In Section 2 we prove Theorems 1(i) and 2(i). In Section 3 we prove part (ii) of the same theorems. In Section 4 we prove Theorem 3, and conclude with a short appendix giving a new proof of a known result on random bipartite graphs.

\section{Groups at density greater than $1 / 2$}

In this section we will use random graphs to understand the relationship between the generators of a random group, and in particular to show that asymptotically almost surely in a random group in a $k$-angular model at density greater than $1 / 2$, all generators are equal.

Definition 2.1. The random bipartite graph $\Gamma(a, b, E(a, b))$ is a graph obtained by sampling uniformly at random from the set of all bipartite graphs $\Gamma$ with parts $V_{1}$ and $V_{2}$ such that $\left|V_{1}\right|=a$, $\left|V_{2}\right|=b$, and $|E(\Gamma)|=E(a, b)$.

The following result is similar to a famed theorem of Erdös and Rényi.

Lemma 2.2 ([12, Theorem 1]). Let $a>0$, let $\varepsilon \in(0,1)$, and let $P\left(a, a, a^{1+\varepsilon}\right)$ denote the probability that a random bipartite graph $\Gamma\left(a, a, a^{1+\varepsilon}\right)$ is connected. Then

$$
\lim _{a \rightarrow \infty} P\left(a, a, a^{1+\varepsilon}\right)=1
$$


The next result follows with only a little work from [5, Theorem 9], where the corresponding result is shown for an Erdös-Rényi random bipartite graph, but for convenience we include a proof in the Appendix to this paper.

Lemma 2.3. Let $d \in(1 / 2,1)$, let $m \geq 1$, and let $\Gamma$ be a $\Gamma\left(n^{m}, n^{m+1}, n^{(2 m+1) d}\right)$ random bipartite graph. Then asymptotically almost surely all vertices in $V_{1}$ are in the same connected component of $\Gamma$.

For the remainder of this paper, let $C_{k, n}$ be the set of cyclically reduced words of length $k$ in $F_{n}$, and $C_{k, n}^{\prime}$ be the set of positive words of length $k$ in $F_{n}$. We now prove a theorem that immediately implies Theorem 2(i).

Theorem 2.4. Let $k \geq 2$ and $d>\frac{1}{2}$. Let $G=\left\langle a_{1}, \ldots, a_{n} \mid R\right\rangle$ be a random group in the positive $(n, k, d)$ model. Asymptotically almost surely the group $G$ is isomorphic to $\mathbb{Z}_{k}$, and $a_{1}=_{G} \cdots={ }_{G} a_{n}$.

Proof. Define a random bipartite graph $\Gamma$ with parts $V_{1}$ and $V_{2}$ as follows. Let $V_{1}=C_{\lfloor k / 2\rfloor, n}^{\prime}$ and let $V_{2}=C_{\lceil k / 2\rceil, n}^{\prime}$. Notice that $C_{k, n}^{\prime}=\left\{x y \mid x \in V_{1}, y \in V_{2}\right\}$. Draw an edge between $x \in V_{1}$ and $y \in V_{2}$ if $x y \in R$. Then $\Gamma$ is a $\Gamma\left(n^{\left\lfloor\frac{k}{2}\right\rfloor}, n^{\left\lceil\frac{k}{2}\right\rceil}, n^{k d}\right)$ random bipartite graph.

Let $\varepsilon=2 d-1>0$. If $k$ is even then $n^{k d}=\left(n^{\frac{k}{2}}\right)^{1+\varepsilon}$, so by Lemma 2.2 a.a.s. this graph is connected. If $k$ is odd then by Lemma 2.3 a.a.s. there exists a connected component of $\Gamma$ spanning $V_{1}$.

Hence in both cases a.a.s. there is a path of even length between any two vertices in $V_{1}$. An edge between $x$ and $y$ corresponds to $x y \in R$, and so $x={ }_{G} y^{-1}$. Hence a path of even length corresponds to equality in $G$, and therefore a.a.s. all elements in $C_{\lfloor k / 2\rfloor, n}^{\prime}$ are equal in $G$. In particular for distinct $a_{i}, a_{j}$, a.a.s. $a_{i}^{\left\lfloor\frac{k}{2}\right\rfloor}={ }_{G} a_{i}^{\left\lfloor\frac{k}{2}\right\rfloor-1} a_{j}$, and so $a_{i}={ }_{G} a_{j}$.

All relators are positive words of length $k$, so a.a.s. $G$ is isomorphic to $\mathbb{Z}_{k}$.

We now wish to show that a.a.s. a random group in the standard $(n, k, d)$ model at density $d>\frac{1}{2}$ contains enough positive words as relators to force the generators to be equal. We first record a well-known probability estimate.

Lemma 2.5 ([13, Corollary 1.1]). Sample without replacement from a finite list $x_{1}, \ldots, x_{M}$. Let $X_{1}, \ldots, X_{m}$ be these samples. Define $S_{m}=\sum_{i=1}^{m} X_{i}, \mu=\frac{1}{M} \sum_{i=1}^{M} x_{i}$. Also let $y_{0}=\min x_{i}$, $y_{1}=\max x_{i}$, and $z_{m}=(m-1) / M$. Then

$$
P\left[S_{m} \geq m \mu+m t\right] \leq \exp \left[-2 m t^{2} /\left(1-z_{m}\right)\left(y_{1}-y_{0}\right)^{2}\right] .
$$

Note that the right hand side of the inequality is less than $\exp \left[-2 m t^{2} /\left(y_{1}-y_{0}\right)^{2}\right]$. We now prove the following, extending from $k=4$ in [7, Lemma 2.9].

Lemma 2.6. Let $k, n \geq 2, d>\frac{1}{2}$, and let $G=\langle X \mid R\rangle$ be a random group in the standard $(n, k, d)$ model. With probability tending to 1 as $n$ tends to $\infty,\left|R \cap C_{k, n}^{\prime}\right| \geq n^{k d^{\prime}}$ for any $\frac{1}{2}<d^{\prime}<d$.

Proof. Randomly selecting $R$ is equivalent to sampling relators from $C_{k, n}$ without replacement. Let $\frac{1}{2}<d^{\prime}<d$. We show that

$P\left(\left|R \cap C_{k, n}^{\prime}\right|<\frac{1}{2^{k}+1}(2 n-1)^{k d}\right)=P\left(\left|R \cap\left(C_{k, n} \backslash C_{k, n}^{\prime}\right)\right| \geq \frac{2^{k}}{2^{k}+1}(2 n-1)^{k d}\right) \rightarrow 0$ as $n \rightarrow \infty$. 
As $n \rightarrow \infty$, with $k$ and $d$ fixed,

$$
\frac{1}{2^{k}+1}(2 n-1)^{k d} \geq \frac{(2 n-1)^{k d-k d^{\prime}}}{2^{k}+1} n^{k d^{\prime}} \geq n^{k d^{\prime}}
$$

and the result will follow.

Consider the following. For relators $r_{1}, \ldots, r_{(2 n-1)^{k d}}$ define the random variable $X_{i}$ by

$$
X_{i}=\left\{\begin{array}{l}
1 \text { if } r_{i} \in C_{k, n} \backslash C_{k, n}^{\prime} \\
0 \text { otherwise }
\end{array}\right.
$$

This equivalent to sampling without replacement from $\left|C_{k, n} \backslash C_{k, n}^{\prime}\right|$ ones and $\left|C_{k, n}^{\prime}\right|$ zeros. Notice that $\left|C_{k, n}\right|<2 n(2 n-1)^{k-1}$, whilst $\left|C_{k, n}^{\prime}\right|=n^{k}$. In the notation of Lemma 2.5,

$$
\begin{gathered}
\mu=\frac{\left|C_{k, n} \backslash C_{k, n}^{\prime}\right|}{\left|C_{k, n}\right|}=1-\frac{\left|C_{k, n}^{\prime}\right|}{\left|C_{k, n}\right|}<1-\frac{n^{k}}{(2 n)^{k}}=1-\frac{1}{2^{k}}, \\
y_{0}=0, y_{1}=1 \text {, and } m=(2 n-1)^{k d} \text {. Hence, letting } t=\frac{2^{k}}{2^{k}+1}-\mu>0, \\
P\left(\left|R \cap C_{k, n} \backslash C_{k, n}^{\prime}\right| \geq \frac{2^{k}}{2^{k}+1}(2 n-1)^{k d}\right)=P_{m}(t) \leq \exp \left[-2(2 n-1)^{k d} t^{2}\right] \rightarrow 0 \text { as } n \rightarrow \infty .
\end{gathered}
$$

Proof of Theorem 1(i). By Lemma 2.6, a.a.s. $G$ has at least $n^{k d^{\prime}}$ positive relators for any $\frac{1}{2}<d^{\prime}<d$. Hence by Theorem 2.4, a.a.s. $a_{i}^{k}={ }_{G} 1$ for all generators $a_{i}$ of $G$, and $a_{i}=a_{j}$ for all $i$ and $j$. Also, a.a.s. there is a cyclic conjugate of a word of the form $a_{i_{1}} \ldots a_{i_{k-1}} a_{i_{k}}^{-1}$ in $R$ - there are at least $k(2 n-2) n^{k-1}$ such cyclic conjugates, and a proof similar to Lemma 2.6 follows. Hence a.a.s $a_{1}^{k-2}={ }_{G} 1$, and so $G$ is isomorphic to a cyclic group of order 2 if $k$ is even, and 1 if $k$ is odd.

\section{Groups at density less than $1 / 2$}

In this section we shall prove Theorems 1(ii) and 2(ii).

We first introduce the diagrams with which we shall be working; our definitions follow [8]. For a set $X$, we write $X^{ \pm}$to denote the (disjoint) union $X \cup X^{-1}$.

Definition 3.1. Let $G=\langle X \mid R\rangle$ be a group. A van Kampen diagram for $G$ is a planar, simplyconnected, finite 2-complex, $A$ such that

i) each 1-cell in $A$ is labelled by an element in $X^{ \pm}$,

ii) if $e$ is an oriented 1-cell with the opposite orientation denoted $e^{-1}$, then the label of $e$ is the inverse of the label of $e^{-1}$,

iii) each 2-cell $D$ of $A$ is oriented, and has a marked start point on $\partial D$. Reading along $\partial D$ from the start point, in the direction given by the orientation, yields a relator $r \in R$. We say that $D$ bears $r$.

If $w \in F_{X}$ is freely reduced, $A$ is a van Kampen diagram for $G$, and there exists a 0-cell in $\partial A$ such that reading clockwise along $\partial A$ and concatenating the labels of the 1-cells in $\partial A, w$ is the word obtained, then $A$ is a van Kampen diagram for $w$, and $w$ is a boundary word for $A$. (If $A$ is a sphere, then $w$ is the empty word.) 
We will write $|A|$ for the number of 2-cells in $A$, and $|\partial A|$ for the number of 1-cells in $\partial A$ (or the length of a boundary word of $A$ ). We will switch freely between the words vertex and 0-cell, edge and 1-cell, and face, region and 2-cell.

Definition 3.2. Let $G$ be a group and $A$ a van Kampen diagram for $G$. The diagram $A$ is unreduced if there exist regions $D_{1}$ and $D_{2}$ in $A$ bearing the same relator with opposite orientations, and with shared edge representing the same letter in the relator (with respect to the marked start points). A van Kampen diagram is reduced if it is not unreduced.

Note that this definition of reduced is slightly weaker than the standard definition, due to the marked start points. It is standard that any unreduced van Kampen diagram can be transformed into a reduced van Kampen diagram without altering the boundary word (as an element of $F(X)$ ).

Definition 3.3. Let $A$ be a van Kampen diagram, with each edge labelled by a generator or its inverse, bearing $N$ distinct relators $r_{i_{1}}, \ldots, r_{i_{N}}$. We construct the abstract van Kampen diagram from $A$ as follows. Each face bearing relator $r_{i_{j}}$ is labelled with the number $j$, we record the orientation of each face, and the starting point of the relator in the face boundary. An abstract van Kampen diagram is reduced if it does not contain two regions with the same relator number that have opposite orientations, share an edge, with the common edge having the same position in the boundary with respect to the marked start position. For an abstract van Kampen diagram, $A$, we write $|\partial A|$ for the number of edges in the boundary of $A$, and $|A|$ for the number of 2-cells in $A$.

The following applies to both van Kampen diagrams and abstract van Kampen diagrams.

Definition 3.4. A spur in a diagram $A$ is an edge such that either its start or end vertex is of degree 1. The diagram $A$ is spurless if it has no spurs. A filament in $A$ is a non-spur edge, $e$, such that for all regions, $D$, in $A$, the intersection $e \cap \partial D=\emptyset$. An edge is non-filamentous if is not a filament. A vertex is exterior if it lies on $\partial A$, and interior if it is not exterior. Similarly, an edge is exterior if it lies on $\partial A$, and interior if it is not exterior.

Notice that if all relators are assumed to be cyclically reduced, then every interior edge is automatically non-filamentous. We shall therefore implicitly make this assumption from now on.

Definition 3.5. An abstract reduced van Kampen diagram $A$ is fulfillable with respect to the presentation $G=\langle X \mid R\rangle$ if there exist relators $r_{i_{1}}, \ldots, r_{i_{N}} \in R$ such that each relator $r_{i_{j}}$ attaches to faces bearing $j$ (respecting start vertex and orientation) and the result is a valid reduced van Kampen diagram for $G$, i.e. there exist letters in $X^{ \pm}$that can label spurs and filaments which gives rise to a reduced van Kampen diagram for $G$. In this case, the relators $r_{i_{j}}$ are said to fulfil $A$. Relators $r_{i_{1}}, \ldots, r_{i_{m}}(m \leq N)$ partially fulfil $A$ if the assignments of $r_{i_{j}}$ to regions bearing $j$ do not produce a contradiction.

Definition 3.6. Let $A$ be an abstract van Kampen diagram. For a face $f$, bearing edge $e$, let $(i(e, f), j(e, f))$ be the tuple where $i(e, f)$ is the relator number $f$ bears, and $j(e, f)$ is the position of $e$ in $\partial f$ with respect to the marked start point. We order the tuples lexicographically, so that $(i(e, f), j(e, f))>\left(i\left(e^{\prime}, g\right), j\left(e^{\prime}, g\right)\right)$ if $i(e, f)>i\left(e^{\prime}, g\right)$ or if $i(e, f)=i\left(e^{\prime}, g\right)$ and $j(e, f)>j\left(e^{\prime}, g\right)$.

If an edge $e$ is interior and incident to faces $f, g$ such that $(i(e, f), j(e, f))>(i(e, g), j(e, g))$, then $e$ belongs to $f$. We do not assign ownership of exterior edges. Note that if $f \neq g$ then $(i(e, f), j(e, f)) \neq(i(e, g), j(e, g))$ since otherwise either $f$ bears the same relator number in the opposite orientation to $g$ and with equivalent start points (and so $A$ is not reduced), or a letter is its own inverse in $F_{n}$. For a face $f$, let $\omega(f)$ be the number of edges that belong to $f$, and for $i=1, \ldots, N$ let

$$
\omega_{i}=\max _{f \text { bearing } i} \omega(f) .
$$


Lemma 3.7. Let $A$ be a reduced abstract van Kampen diagram with relator numbers $\{1, \ldots, N\}$ and with all faces of boundary length $k$. For $i=1, \ldots, N$, let $p_{i}$ be the probability that $i$ randomly chosen cyclically reduced words of length $k$ partially fulfil $A$ (as relator numbers $1, \ldots, i$ ), and let $p_{0}=1$. Then for $i=1, \ldots, N$,

$$
\frac{p_{i}}{p_{i-1}} \leq(2 n-1)^{-\omega_{i}} \frac{2 n-1}{2 n-2}
$$

Proof. Let $1 \leq i \leq N$. Let us assume this is true for $p_{1}, \ldots, p_{i-1}$, and assume that we have chosen relators $w_{1}, \ldots, w_{i-1}$ that partially fulfil $A$. Now suppose that we have chosen $0 \leq l<k$ letters of the relator $w_{i}$, and let $f$ be a face of $A$ bearing relator number $i$. We now choose the letter corresponding to an edge $e$ of $f$.

If $e$ belongs to $f$, then there exists another face, $g$, with $(i(e, g), j(e, g))<(i(e, f), j(e, f))$. This means that either the edge appears in some word $w_{j}$ for $j<i$, and so the label of $e$ is already fixed, or it appears as an earlier edge in a face bearing the same relator as $f$ (and so as an earlier edge in $f$ ), and therefore the label of $e$ is also fixed. We choose letters to obtain a cyclically reduced word: there are $2 n$ choices for the first, $2 n-1$ for the middle $k-2$ letters, and $2 n-1$ or $2 n-2$ choices for the last letter. Hence the probability of a randomly chosen letter being valid is less than or equal to $\frac{1}{2 n} \leq \frac{1}{2 n-1}$ if this is the first letter, $\frac{1}{2 n-1}$ for the middle $k-2$ letters, and $\frac{1}{2 n-1}$ or $\frac{1}{2 n-2}$ for the final letter.

If $e$ does not belong to $f$, then the probability that a randomly chosen label for $e$ partially fulfils $A$ is at most 1.

So having chosen $i-1$ random words partially fulfilling $A$, the probability that the next random word partially fulfils $A$ is less than or equal to $(2 n-1)^{-(\omega(f)-1)}(2 n-2)^{-1}$ for all $f$ bearing $i$. Hence the probability, $p_{i}$, of $i$ randomly chosen words partially fulfilling $A$ satisfies

$$
p_{i} \leq p_{i-1} \min _{f \text { bearing } i}\left\{(2 n-1)^{-\omega(f)}\right\} \frac{2 n-1}{2 n-2}=p_{i-1}(2 n-1)^{-\omega_{i}} \frac{2 n-1}{2 n-2},
$$

and the result follows.

We proceed by evaluating the probability that an abstract reduced van Kampen diagram satisfies the linear isoperimetric inequality or the probability that it can be fulfilled if it does not. The following lemma is a slightly more precise version of [9, Proposition 58].

Lemma 3.8. Let $n, k \geq 2, d<\frac{1}{2}$, and $\varepsilon>0$. Let $G=\langle X \mid R\rangle$ be a random group in the standard $(n, k, d)$ model. Any spurless abstract reduced van Kampen diagram, A, for $G$ either satisfies

$$
|\partial A| \geq k|A|\left(1-2 d-2 \varepsilon-\frac{2}{k}\left(1-\log _{(2 n-1)}(2 n-2)\right)\right)
$$

or the probability it is fulfillable in $G$ is at most $(2 n-1)^{-\varepsilon k}$.

Proof. Let $A$ be a spurless abstract reduced van Kampen diagram, let $N$ be the number of distinct relators in $A$, so that $N \leq|A|$, and let $m_{i}$ be the number of faces bearing relator number $i$. We can assume without loss of generality that the $m_{i}$ are non-increasing.

Now, $k|A|$ counts each interior edge twice, and each non filamentous exterior edge once, but does not count any filaments. Also,

$$
\sum_{f \text { face of } A} \omega(f)
$$

counts each interior edge once. So

$$
|\partial A| \geq k|A|-2 \sum_{f \text { face of } A} \omega(f) .
$$


Let $p_{i}$ be as in Lemma 3.7, and for brevity let $\alpha=2 n-1$. Then $\frac{p_{i}}{p_{i-1}} \leq \alpha^{-\omega_{i}} \frac{\alpha}{\alpha-1}$. Therefore

$$
-\omega_{i} \geq \log _{\alpha} p_{i}-\log _{\alpha} p_{i-1}+\log _{\alpha}(\alpha-1)-1 \text {. }
$$

Hence

$$
\begin{aligned}
|\partial A| & \geq k|A|-2 \sum_{f \text { face of } A} \omega(f) \\
& \geq k|A|-2 \sum_{i=1}^{N} m_{i} \omega_{i} \\
& \geq k|A|+2\left(\sum_{i=1}^{N} m_{i}\left(\log _{\alpha} p_{i}-\log _{\alpha} p_{i-1}+\log _{\alpha}(\alpha-1)-1\right)\right) .
\end{aligned}
$$

Note that $p_{0}=1$, so

$$
\sum_{i=1}^{N} m_{i}\left(\log _{\alpha} p_{i}-\log _{\alpha} p_{i-1}\right)=\left(\sum_{i=1}^{N-1}\left(m_{i}-m_{i+1}\right) \log _{\alpha} p_{i}\right)+m_{N} \log _{\alpha} p_{N} .
$$

Therefore, as $\sum_{i=1}^{N} m_{i}=|A|$,

$$
\begin{aligned}
|\partial A| & \geq k|A|+2\left(\sum_{i=1}^{N-1}\left(m_{i}-m_{i+1}\right) \log _{\alpha} p_{i}\right)+2 m_{N} \log _{\alpha} p_{N}-2|A|\left(1-\log _{\alpha}(\alpha-1)\right) \\
& =|A|\left(k-2\left(1-\log _{\alpha}(\alpha-1)\right)\right)+2\left(\sum_{i=1}^{N-1}\left(m_{i}-m_{i+1}\right) \log _{\alpha} p_{i}\right)+2 m_{N} \log _{\alpha} p_{N} .
\end{aligned}
$$

Let $P_{i}$ be the probability that there exist $i$ relators in $R$ partially fulfilling $A$. Notice that $P_{i} \leq|R|^{i} p_{i}$, so that $P_{i} \leq \alpha^{i d k} p_{i}$, and hence $\log _{\alpha} p_{i} \geq \log _{\alpha} P_{i}-i d k$. Substituting, we get

$|\partial A| \geq|A|\left(k-2\left(1-\log _{\alpha}(\alpha-1)\right)\right)+2\left(\sum_{i=1}^{N-1}\left(m_{i}-m_{i+1}\right)\left(\log _{\alpha} P_{i}-i d k\right)\right)+2 m_{N}\left(\log _{\alpha} P_{N}-N d k\right)$.

Now,

$$
\sum_{i=1}^{N-1}\left(m_{i}-m_{i+1}\right) i+m_{N} N=\sum_{i=1}^{N} m_{i}=|A| .
$$

so we can rearrange the previous expression to get

$$
|\partial A| \geq|A|\left(k-2\left(1-\log _{\alpha}(\alpha-1)\right)\right)-2 d k|A|+2\left(\sum_{i=1}^{N-1}\left(m_{i}-m_{i+1}\right) \log _{\alpha} P_{i}\right)+2 m_{N} \log _{\alpha} P_{N},
$$

Now let $P=\min _{i} P_{i}$. Then from $m_{i}-m_{i+1} \geq 0$ and $\log _{\alpha} P_{i} \geq \log _{\alpha} P$ we deduce that

$$
\begin{aligned}
|\partial A| & \geq|A|\left(k-2 d k-2\left(1-\log _{\alpha}(\alpha-1)\right)\right)+2\left(\sum_{i=1}^{N-1}\left(m_{i}-m_{i+1}\right) \log _{\alpha} P\right)+2 m_{N} \log _{\alpha} P \\
& \geq|A|\left(k-2 d k-2\left(1-\log _{\alpha}(\alpha-1)\right)\right)+2 m_{1} \log _{\alpha} P \\
& \geq|A| k\left(1-2 d-(2 / k)\left(1-\log _{\alpha}(\alpha-1)\right)\right)+2|A| \log _{\alpha} P .
\end{aligned}
$$


So

$$
P \leq \alpha^{\frac{1}{2}\left(\frac{|\partial A|}{|A|}-k\left(1-2 d-\frac{2}{k}\left(1-\log _{\alpha}(\alpha-1)\right)\right)\right) .}
$$

Now it is immediate that $P(A$ fulfillable $) \leq P$. If

$$
\frac{1}{2}\left(\frac{|\partial A|}{|A|}-k\left(1-2 d-\frac{2}{k}\left(1-\log _{\alpha}(\alpha-1)\right)\right)\right) \leq-\varepsilon k
$$

then

$$
P(A \text { fulfillable }) \leq P \leq \alpha^{-\varepsilon k}=(2 n-1)^{-\varepsilon k} .
$$

If Equation(1) does not hold, then

$$
|\partial A|>k|A|\left(1-2 d-2 \varepsilon-\frac{2}{k}\left(1-\log _{(2 n-1)}(2 n-2)\right)\right)
$$

and the result follows.

Setting $\delta=\frac{\varepsilon}{2}$, letting $n$ be large enough so that $\left.\frac{2}{k}\left(1-\log _{(2 n-1)}(2 n-2)\right)\right) \leq \varepsilon$, and substituting $\delta$ for $\varepsilon$ into Lemma 3.8 yields the following slightly simpler statement.

Lemma 3.9. Let $k, n \geq 2, d<\frac{1}{2}$, and $\varepsilon>0$. Let $G=\langle X \mid R\rangle$ be a random group in the standard $(n, k, d)$ model. For large enough $n$ any abstract reduced van Kampen diagram, $A$, for $G$ either satisfies $|\partial A| \geq k(1-2 d-2 \varepsilon)|A|$ or the probability it is fulfillable in $G$ is less than $(2 n-1)^{-\frac{\varepsilon}{2} k}$.

Lemma 3.9 gives us a bound on the probability that a single abstract spurless van Kampen diagram fails to satisfy a given isoperimetric inequality. To generalise the result to all such diagrams, we first count them. The following proof is very slightly corrected from [8, p614].

Lemma 3.10. Let $m \in \mathbb{N}$ and $G$ be a group with relators all of length $k$. The number $N(m, k)$ of abstract spurless reduced van Kampen diagrams for $G$ with at most $m$ faces is bounded above by $G(m)\left(2 m k^{4}\right)^{m}$, where $G(m)$ is a constant depending only on $m$.

Proof. Let $D$ be an abstract spurless reduced van Kampen diagram for $G$, with boundary length $\lambda \geq 0$ and $t$-cells.

If $t=1$, then $\lambda \geq 1$. Such a diagram has one choice for relator number, 2 choices for orientation, and a single choice (up to equivalence) for the start point of the relator.

If $\lambda=0$ and $t=2$, then there are two choices for relator numbers, four choices of orientation of the two 2-cells, and up to $k$ choices for the distance between the two labelled start points.

If $t>2$, or if $t=2$ and $\lambda \geq 1$, then $D$ can be thought of as a connected planar graph with $t-1$ or $t$ faces (including the external face, if any), with vertices all of degree at least three, along with some extra information. By Euler's formula, any such graph with at least 2 faces and at most $t$ faces has at most $3 t$ edges. Each edge can have label length between 1 and $k$. Each non-external face can have one of two orientations, one of $k$ start points for the relator, and one of at most $t$ choices of relator. So there are at most $(2 k t)^{t}$ choices for the decoration of all of the faces.

For $m>1$, let $G(m)-1$ be the number of connected planar graphs with at most $m$ faces and with all vertices of degree at least 3 . Then there are at most $(G(m)-1)\left(2 m k^{4}\right)^{m}$ spurless reduced abstract van Kampen diagrams with at least two regions (or at least three regions when spherical) and at most $m$ regions. Therefore $N(m, k) \leq(G(m)-1)\left(2 m k^{4}\right)^{m}+2+8 k \leq G(m)\left(2 m k^{4}\right)$.

The following immediately implies the hyperbolicity claims in Theorems 1(i) and 2(i). 
Theorem 3.11. Let $k, n \geq 2, d<\frac{1}{2}$, and let $G=\langle X \mid R\rangle$ be a random group in an $(n, k, d)$ model. Let $\varepsilon=(1-2 d) / 8$, and $C=(1-2 d-2 \varepsilon)$. Asymptotically almost surely any van Kampen diagram, $A$, for $G$ satisfies $|\partial A| \geq k(C-\varepsilon)|A|$, and so $G$ is hyperbolic.

Proof. We consider the standard model first. By Lemma 3.9, any spurless abstract reduced van Kampen for $G$ either satisfies $|\partial A| \geq k C|A|$ or the probability it is fulfillable is less than $(2 n-1)^{-\frac{\varepsilon}{2} k}$.

The probability that any spurless reduced van Kampen diagram for $G$ with at most $m$ faces does not satisfy the above inequality is less than $G(m)\left(2 m k^{4}\right)^{m}(2 n-1)^{-\frac{\varepsilon}{2} k} \rightarrow 0$ as $n \rightarrow \infty$. So a.a.s. any spurless reduced van Kampen diagram for $G$ with at most $m$ faces satisfies $|\partial A| \geq k C|A|$, and so the same inequality holds for any reduced van Kampen diagram $A$ for $G$ with at most $m$ faces. Hence by $[10$, Theorem 8], a.a.s. any reduced van Kampen diagram in $G$ satisfies $|\partial A| \geq(C-\varepsilon) k|D|$, and so $G$ is hyperbolic.

For the positive model, we argue as in Lemma 3.8, but replace Lemma 3.7 with $p_{i} \leq p_{i-1} n^{-\omega_{i}}$, to deduce that any abstract reduced van Kampen diagram $A$ for $G$ either satisfies $|\partial A| \geq k|A|(1-$ $2 d-2 \varepsilon)$ or the probability it is fulfillable in $G$ is less than $n^{-\varepsilon k}$. The result then follows exactly as for the standard model.

Finally, we show that at density less than $1 / 2$ our groups are infinite and torsion-free, and looking ahead to the next section we consider freeness.

Proposition 3.12. Let $k \geq 2$, and let $G$ be a random group in an $(n, k, d)$ model. If $0<d<\frac{1}{2}$ then asymptotically almost surely $G$ is infinite and torsion-free. If $\frac{1}{k}<d<\frac{1}{2}$ then asymptotically almost surely $G$ is not isomorphic to a free group.

Proof. We showed in Theorem 3.11 that at density $d<1 / 2$ the group $G$ satisfies a linear isoperimetric inequality with additive constant zero, which implies that there are no van Kampen diagrams with boundary length 0 . Our definitions of van Kampen diagrams were sufficiently general that this implies that the Cayley 2-complex is aspherical. This implies that $G$ has cohomological dimension at most 2 , and so is torsion free.

The only finite group that is torsion-free is the trivial group, however the Euler characteristic of $G$ is a.a.s. equal to $1-n+(2 n-1)^{d k}$ in the standard model and $1-n+n^{d k}$ in the positive model. For $1 / 2>d>1 / k$ this characteristic is a.a.s. greater than one and grows arbitrarily large with $n$. Conversely, the trivial group has Euler characteristic 1 and a free group of rank $\ell$ has Euler characteristic $1-\ell$.

This concludes the proof of Theorems 1(ii) and 2(ii).

\section{Groups at density less than $1 / k$}

In this section we prove that a group $G$ in either $k$-angular model is asympotically almost surely free if the density is less than $1 / k$. The fact that this bound is tight follows from Proposition 3.12.

We start by proving a sufficient condition on the presentation complex for a group to be free.

Definition 4.1. Let $G=\langle X \mid R\rangle$ be a group presentation. We construct the presentation complex, $\mathcal{P}$, for $G$ as follows. Take a single vertex, $v$, as the only 0 -cell. Take as the 1-cells oriented loops $x_{i}$ at $v$ for each $x_{i} \in X$. Take a 2-cell for each relator, $r_{i}$, with the boundary mapped to the succession of appropriately oriented 1-cells $x_{i_{1}}^{\epsilon_{1}}, \ldots, x_{i_{n}}^{\epsilon_{n}}$ with $\epsilon \in\{ \pm 1\}$ such that $r_{i}=x_{i_{1}}^{\epsilon_{1}} \ldots x_{i_{n}}^{\epsilon_{n}}$.

It is standard that $\Pi_{1}(\mathcal{P}) \cong G$. We remark that in an $(n, k, d)$ model we can define the closed 2 -cells of $\mathcal{P}$ to be isometric with the closed unit $k$-gon in Euclidean space, and assign the path metric to $\mathcal{P}$ to turn $\mathcal{P}$ into a metric complex. 
The next set of definitions are based on those of [11].

Definition 4.2. Let $\mathcal{C}$ be a path-connected 2-complex such that all 2-cells have even boundary length. We construct a labelled undirected graph, $\Gamma_{\mathcal{C}}$, called the antipodal graph for $\mathcal{C}$. Take as the vertices the set of 1-cells in $\mathcal{C}$. For each 2-cell $F$, and for each pair $v_{1}, v_{2}$ of (not necessarily distinct) 1-cells that are antipodal on $\partial(F)$, add an edge $\left\{v_{1}, v_{2}\right\}$ to $\Gamma_{\mathcal{C}}$ labelled $F$. We say that $F$ is the 2-cell containing the edge $\left\{v_{1}, v_{2}\right\}$.

If $\mathcal{C}$ is a metric complex, then there is a map, $\phi: \Gamma_{\mathcal{C}} \rightarrow \mathcal{C}$, as follows. The map $\phi$ sends each vertex of $\Gamma_{\mathcal{C}}$ to the midpoint of the corresponding 1-cell of $\mathcal{C}$, and sends each edge $\left\{v_{1}, v_{2}\right\}$ of $\Gamma_{\mathcal{C}}$, labelled $F$, to a (non self-intersecting) path in $F \backslash \partial F$ joining $\phi\left(v_{1}\right)$ and $\phi\left(v_{2}\right)$.

Definition 4.3. Let $\mathcal{C}$ be a path-connected 2-complex such that all 2-cells have odd boundary length. Form the halved complex $H_{\mathcal{C}}$ of $\mathcal{C}$ as follows. Replace each 1-cell, $e$, in $\mathcal{C}$, by two 1-cells $e_{1}$ and $e_{2}$, meeting at a 0 -cell. The edge $e$ is the precursor of $e_{1}$ and $e_{2}$. The antipodal graph for $H_{\mathcal{C}}$ is the halved antipodal graph of $\mathcal{C}$; we write $\Gamma_{\mathcal{C}}^{H}:=\Gamma_{H_{\mathcal{C}}}$.

If $\mathcal{C}$ is metric, then there is a map $\phi^{H}: \Gamma_{\mathcal{C}}^{H} \rightarrow \mathcal{C}$, as follows. The map $\phi$ sends each vertex $v$ to the midpoint of the precursor of $v$ in $\mathcal{C}$, and sends each edge $\left\{v_{1}, v_{2}\right\}$ of $\Gamma_{\mathcal{C}}^{H}$, labelled $F$, to a (non self-intersecting) path in $F \backslash \partial F$ between $\phi\left(v_{1}\right)$ and $\phi\left(v_{2}\right)$.

Notice that the graph $\Gamma_{\mathcal{C}}^{H}$ consists of two disjoint components.

Definition 4.4. Let $\mathcal{C}$ be a path-connected 2-complex such that the boundary lengths of the 2-cells are either all odd, or all even. Let $\Gamma$ be $\Gamma_{\mathcal{C}}$ in the even case, or $\Gamma_{\mathcal{C}}^{H}$ in the odd case, and let $\varphi=\phi$ in the even case, or $\varphi=\phi^{H}$ in the odd case. A hypergraph $\Lambda$ of $\Gamma$ is a connected component of $\Gamma$. We also refer to $\varphi(\Lambda)$ as a hypergraph of $\mathcal{C}$, and if $\Lambda$ is a tree, then we will also refer to $\varphi(\Lambda)$ as a tree.

Theorem 4.5. Let $G=\langle X \mid R\rangle$ be a finite presentation with all relators of length $k$, such that all hypergraphs in the presentation complex $\mathcal{P}$ of $G$ are trees. Then $G$ is a free group.

Proof. Let $\Gamma=\Gamma_{\mathcal{P}}$ when $k$ is even, and $\Gamma=\Gamma_{\mathcal{P}}^{H}$ when $k$ is odd. We show by induction on the number of edges of $\Gamma$ that $G$ is free.

The result is clear when $\Gamma$ has no edges, as then $\mathcal{P}$ is just a bouquet of circles. Assume therefore that $\Gamma$ contains at least $i \geq 1$ edges. Let $\Lambda$ be a connected component in $\Gamma$ containing at least one edge. Then $\Lambda$ is a tree, and so contains at least one vertex of valency 1 , which must correspond to a 1 -cell in $\mathcal{P}$ which is on the boundary of a single 2-cell. This means that there is a letter $a \in X$ which occurs in a single relator $r \in R$, and we can apply a Tietze transformation to the presentation which replaces $X$ by $X^{\prime}=X \backslash\{a\}$ and replaces $R$ by $R^{\prime}=R \backslash\{r\}$ (all other relators are unchanged). Then $G \cong\left\langle X^{\prime} \mid R^{\prime}\right\rangle$.

Let $\mathcal{P}^{\prime}$ be the presentation complex of this new presentation. If $k$ is even then $\Gamma^{\prime}=\Gamma_{\mathcal{P}^{\prime}}$ has vertex set $X \backslash a$, and edge set a subset of $E(\Gamma)$ with precisely $k / 2 \geq 1$ edges removed. If $k$ is odd then $\Gamma^{\prime}=\Gamma_{\mathcal{P}^{\prime}}$ has vertex set a subset of $X$ with the two precusors of $a$ removed, and edge set a subset of $E(\Gamma)$ with precisely $k>1$ edges removed. Thus all connected components of $\Gamma^{\prime}$ are trees, and each such component embeds in $\mathcal{P}$. Hence the result follows by induction.

We now introduce two further variants on van Kampen diagrams, which will give us a necessary condition for all hypergraphs in a presentation complex to be trees.

Definition 4.6. Let $G=\langle X \mid R\rangle$ be a presentation. A relator diagram for $G$ is defined in exactly the same way as a van Kampen diagram, except that we permit the complex to be annular, and we permit it to be homeomorphic to a Möbius strip. 
An abstract relator diagram is the 2-complex obtained from a relator diagram $A$ in the same way as an abstract van Kampen diagram is obtained from a van Kampen diagram. That is, for each 2-cell $f$ bearing relator $r_{i_{j}}$, label the 2-cell with the number $j$, remember only the starting point of the relator and the orientation of the relator on $\partial f$. The definition of relators fulfilling an abstract relator diagram follows as for an abstract reduced van Kampen diagram.

Definition 4.7. Let $G=\langle X \mid R\rangle$ be a finite presentation with all relators of length $k$, and let $D$ be a relator diagram for $G$. We say $D$ satisfies Condition $\dagger(2)$ if $D$ has exactly two 2-cells $F_{1}$ and $F_{2}$, bearing different relator numbers, and $\partial F_{1}$ and $\partial F_{2}$ share exactly two 1-cells. For $\ell>2$, we say $D$ satisfies Condition $\dagger(\ell)$ if

i) $D$ has exactly $\ell$ 2-cells, $F_{1}, \ldots, F_{\ell}$, each bearing a distinct relator, and $(k-1) \ell$ 1-cells,

ii) for $2 \leq j \leq \ell-1, \partial F_{j}$ shares a single 1-cell with $\partial F_{j-1}$, another with $\partial F_{j+1}$, and none with any other 2-cells,

iii) and $\partial F_{1}$ shares a single 1-cell with $\partial F_{\ell}$.

Thus the 2-cells $F_{1}, \ldots, F_{\ell}$ form an annulus, or a Möbius strip, or the neighborhood of an internal vertex in $D$.

Lemma 4.8. Let $G=\langle X \mid R\rangle$ be a finite presentation with all relators of length $k$, and let $\mathcal{P}$ be the presentation complex of $G$. Suppose $G$ has no relator diagrams satisfying Condition $\dagger(\ell)$ for any $\ell \geq 2$, and is such that no letter in $X^{ \pm}$(or its inverse) appears more than once in any relator. Then all hypergraphs in $\mathcal{P}$ are trees.

Proof. We prove this by contradiction. If $k$ is even, then let $\Gamma=\Gamma_{\mathcal{P}}$ and $\varphi=\phi$. If $k$ is odd, then let $\Gamma=\Gamma_{\mathcal{P}}^{H}$ and $\varphi=\phi^{H}$.

Suppose there exists a hypergraph $\Lambda$ in $\mathcal{P}$ such that $\varphi(\Lambda)$ contains a circuit. This means that there is a path $e_{1}, \ldots, e_{m}$ of edges in $\Gamma$, with $m \geq 1$, such that $\varphi$ is not injective when restricted to this path. We can assume that this path is of minimal length, so that $e_{i} \neq e_{j}$ for $i \neq j$ : this may mean that the path is not a cycle in $\Gamma$.

If $m=1$, then the edge $e_{1}$ is a loop in $\Gamma$, since $\varphi\left(e_{1}\right)$ can only self-intersect at its end-points. However this means that a letter appears more than once in a relator, a contradiction.

For $m \geq 2$, form the following relator diagram $D$. Pick as the first 2-cell $F_{1}$ the (unique) 2-cell which contains $e_{1}$. Now for $2 \leq i \leq m$, pick $F_{i}$ as the 2-cell containing $\varphi\left(e_{i}\right)$. The minimality of $m$ ensures that each $F_{i}$ is distinct, except possibly $F_{m}=F_{1}$ when $m \geq 3$, and it is clear that $D$ satisfies the three requirements of Condition $\dagger(m)$ if $F_{m} \neq F_{1}$ and $\dagger(m-1)$ otherwise. By assumption such a relator diagram does not exist, and so such a $\Lambda$ does not exist.

We now consider the first necessary condition for freeness in Lemma 4.8, but first we prove a small combinatorial lemma.

Lemma 4.9. Let $a, b$, and $c$ be positive integers. Then

i) if $a>b>c$ then $\frac{\left(\begin{array}{l}a-c \\ b-c\end{array}\right)}{\left(\begin{array}{l}a \\ b\end{array}\right)} \leq\left(\frac{b}{a}\right)^{c}$,

ii) if $a>b$ and $a>c$ then $\frac{\left(\begin{array}{c}a-c \\ b\end{array}\right)}{\left(\begin{array}{c}a \\ b\end{array}\right)} \leq\left(\frac{a-c}{a}\right)^{b}=\left(1-\frac{c}{a}\right)^{b}$. 
Proof. We calculate

$$
\frac{\left(\begin{array}{l}
a-c \\
b-c
\end{array}\right)}{\left(\begin{array}{l}
a \\
b
\end{array}\right)}=\frac{(a-c) !}{(a-b) !(b-c) !} \cdot \frac{b !(a-b) !}{a !}=\frac{(a-c) !}{a !} \cdot \frac{b !}{(b-c) !}=\left(\frac{b}{a}\right)\left(\frac{b-1}{a-1}\right) \cdots\left(\frac{b-c+1}{a-c+1}\right) .
$$

Notice that for $x<y$, and $i>0$,

$$
\frac{x}{y}>\frac{x-i}{y-i}
$$

and part $i$ ) follows. Similarly, we observe

$$
\frac{\left(\begin{array}{c}
a-c \\
b
\end{array}\right)}{\left(\begin{array}{c}
a \\
b
\end{array}\right)}=\frac{(a-c)(a-c-1) \cdots(a-b-c+1)}{a(a-1) \cdots(a-b+1)}=\left(\frac{a-c}{a}\right)\left(\frac{a-c-1}{a-1}\right) \cdots\left(\frac{a-b-c+1}{a-b+1}\right),
$$

and again the result follows.

Lemma 4.10. Let $k \geq 2$, let $0<d<\frac{1}{k}$, and let $G=\langle X \mid R\rangle$ be a random group in an $(n, k, d)$ model. Let $\ell \geq 2$, and let $P_{\ell}$ denote the probability that an abstract relator diagram which gives rise to a relator diagram satisfying Condition $\dagger(\ell)$ is fulfillable in $G$.

There exists a constant $c:=c(d, k)<0$, depending on $d, k$ and the choice of model, such that for sufficiently large $n$, the value of $P_{\ell}$ is at most $(2 n-1)^{c \ell}$ for the standard model, and at most $n^{c \ell}$ for the positive model.

Proof. First consider the standard model. Let $D_{\ell}$ be an abstract relator diagram which gives rise to a relator diagram satisfying Condition $\dagger(\ell)$.

Let $R_{\ell}$ denote the set of distinct $\ell$-tuples of cyclically reduced words fulfilling $D_{\ell}$ : we first determine $\left|R_{\ell}\right|$. There are $(k-1) \ell$-cells in $D_{\ell}$, the first of which may be labelled freely whilst the rest have at most $2 n-1$ possible labels. Hence $\left|R_{\ell}\right| \leq(2 n)(2 n-1)^{(k-1) \ell-1}$, and so for any $\delta_{1}>0$, for $n$ sufficiently large we can bound

$$
\left|R_{\ell}\right| \leq(2 n-1)^{\left(k-1+\delta_{1}\right) \ell} .
$$

For $\alpha \in R_{\ell}$, let $P_{\alpha}$ be the probability that $\alpha \subseteq R$; that is, the probability that these particular $\ell$ cyclically reduced words are in $R$. Then

$$
P_{\ell} \leq \sum_{\alpha \in R_{\ell}} P_{\alpha}
$$

Recall that $C_{k, n}$ denotes the set of cyclically reduced words of length $k$ in $F_{n}$, and $\left|C_{k, n}\right|$ is larger than $(2 n-1)^{k d}$, which is larger than $\ell$ for $n$ sufficiently large. Hence, by Lemma 4.9(i)

$$
P_{\alpha}=\frac{\left(\begin{array}{c}
\left|C_{k, n}\right|-\ell \\
(2 n-1)^{k d}-\ell
\end{array}\right)}{\left(\begin{array}{c}
\left|C_{k, n}\right| \\
(2 n-1)^{k d}
\end{array}\right)}<\left(\frac{(2 n-1)^{k d}}{\left|C_{k, n}\right|}\right)^{\ell} .
$$

Now, for any $\delta_{2}>0$, for $n$ sufficiently large $\left|C_{k, n}\right|>(2 n-1)^{k-\delta_{2}}$. Therefore, for $n$ sufficiently large we can bound $P_{\alpha}<(2 n-1)^{\left(k d-k+\delta_{2}\right) \ell}$, and so

$$
P_{\ell} \leq\left|R_{\ell}\right| P_{\alpha}<(2 n-1)^{\left(k-1+\delta_{1}\right) \ell}(2 n-1)^{\left(k d-k+\delta_{2}\right) \ell}=(2 n-1)^{\left(k d-1+\delta_{1}+\delta_{2}\right) \ell},
$$

for all $\delta_{1}, \delta_{2}>0$.

Since $k d-1<0$, we can choose $\delta_{1}, \delta_{2}>0$ such that $c=c(d, k)=k d-1+\delta_{1}+\delta_{2}<0$. We then conclude that for $n$ sufficiently large we can bound $P_{\ell} \leq(2 n-1)^{c \ell}$, as required.

The proof for the positive model is similar but easier: $\left|R_{\ell}\right|=n^{(k-1) \ell}$ and $P_{\alpha}<n^{k \ell(d-1)}$, so $P_{\ell} \leq n^{(k d-1) \ell}$, and we can set $c=c(d, k)=k d-1<0$. 
Theorem 4.11. Let $k \geq 2,0<d<\frac{1}{k}$, and $G=\langle X \mid R\rangle$ be a random group in an $(n, k, d)$ model. Asymptotically almost surely $G$ has no relator diagrams satisfying Condition $\dagger(\ell)$ for any $\ell \geq 2$.

Proof. First we show that the number of abstract relator diagrams giving rise to a relator diagram for $G$ satisfying Condition $\dagger(\ell)$ is at most $(2 k)^{\ell}$. Due to Condition $\dagger(\ell)$, every face of such an abstract relator diagram is labelled by a different number. Hence there is (up to equivalence) only one way to choose the labels. There are 2 choices of orientation for each face, and $k$ choices of start point for each relator. Hence there are at most $(2 k)^{\ell}$ such abstract relator diagrams.

Now let $m=2 n-1$ for the standard model, and $m=n$ for the positive model. By Lemma 4.10, there exists a constant $c<0$ such that for $m$ sufficiently large the probability that a relator diagram satisfying Condition $\dagger(\ell)$ exists for $G$ is at most $(2 k)^{\ell} m^{c \ell}$. Since $2 k m^{c}$ tends to 0 as $m \rightarrow \infty$, the probability that such a diagram exists in $G$ for any $\ell$ is at most

$$
\sum_{\ell=2}^{\infty}\left(2 k m^{c}\right)^{\ell} \leq \sum_{\ell=0}^{\infty}\left(2 k m^{c}\right)^{\ell}-1=\frac{1}{1-2 k m^{c}}-1
$$

which tends to 0 as $m$ tends to $\infty$.

Proof of Theorem 3. First we show that asymptotically almost surely there are no repeated letters from $X$ in any relator of $R$. Consider the standard $(n, k, d)$ model. The probability that a fixed letter (or its inverse) appears twice in a fixed relator is at most $\frac{k(k-1)}{2} \frac{4}{(2 n-2)^{2}}$, since at each position in the relator we have at least $2 n-2$ choices for the letter. The set $X$ has size $n$, and there are $(2 n-1)^{d k}$ relators, so the probability that any letter appears more than once in a relator is at most

$$
\frac{k(k-1) 2 n(2 n-1)^{d k}}{(2 n-2)^{2}} \rightarrow 0 \text { as } n \rightarrow \infty .
$$

The proof for the positive model is similar but easier.

By Theorem 4.11, asymptotically almost surely $G$ has no relator diagrams satisfying Condition $\dagger(\ell)$ for any $\ell \geq 2$, so by Lemma 4.8, asymptotically almost surely all hypergraphs in the presentation complex $\mathcal{P}$ of $G$ are embedded trees. The result now follows from Theorem 4.5.

\section{Appendix: proof of Lemma 2.3}

We thank Louis Theran for sketching out some of the ideas in this section to us.

Definition 5.1. An Erdös-Rényi random bipartite graph $\Gamma(a, b, p)$ is a bipartite graph with $\left|V_{1}(\Gamma)\right|=$ $a,\left|V_{2}(\Gamma)\right|=b$, and with each edge added with probability $p$.

First we establish a lower bound of the degree of each vertex in $V_{1}$.

Lemma 5.2. Let $d>\frac{1}{2}$, let $m \geq 1$, fix $0<\varepsilon<d-\frac{1}{2}$, and let $p=n^{(2 m+1)(d-1)}$. With probability tending to 1 as $n \rightarrow \infty$, all vertices in $V_{1}$ in $\Gamma\left(n^{m}, n^{m+1}, p\right)$ have degree at least $M_{n, \varepsilon}=n^{\frac{1}{2}+\varepsilon(2 m+1)}$.

Proof. Fix $v \in V_{1}$. The degree $d(u)$ of a vertex $u \in V_{1}$ satisfies $d(u) \sim B\left(n^{m+1}, p\right)$. Furthermore, as our graph is bipartite, the degrees of these vertices are independent, so the probability $P_{k}$ that there exists a vertex in $V_{1}$ of degree less than $M_{n, \varepsilon}$ is $P_{\varepsilon}=n^{m} P\left(d(v)<M_{n, \varepsilon}\right)$. Let $\lambda=d-1 / 2$, so that $\lambda>\varepsilon$. Notice that $M_{n, \varepsilon}=n^{1 / 2+\varepsilon(2 m+1)}$, whilst the expected value of $d(v)$ is $n^{m+1} p=n^{1 / 2+\lambda(2 m+1)}$, so we can use the multiplicative lower tail form of Chernoff's inequality to bound

$$
P\left(d(v)<M_{n, \varepsilon}\right)=P\left(d(v) \leq M_{n, \varepsilon}-1\right) \leq \exp \left(-\frac{\left(n^{m+1} p-\left(M_{n, \varepsilon}-1\right)\right)^{2}}{2 n^{m+1} p}\right),
$$


and so $P_{\varepsilon} \leq n^{m} \exp \left(-\frac{\left(n^{m+1} p-\left(M_{n, \varepsilon}-1\right)\right)^{2}}{2 n^{m+1} p}\right)$, which tends to 0 as $n \rightarrow \infty$.

The following standard result is proved by by approximating the binomial distribution $\operatorname{Bin}(t, p)$ by the normal distribution $N(t p, t p(1-p))$.

Lemma 5.3. Let $X \sim \operatorname{Bin}(t, p)$, such that $p$ is a function of $t$, and $t p=\omega(1)$. Then with probability tending to 1 as $t$ tends to $\infty, X=(1+o(1))$ tp.

Lemma 5.4. Let $d>\frac{1}{2}, m \geq 1,0<\varepsilon<d-\frac{1}{2}, M_{n, \varepsilon}=n^{\frac{1}{2}+\varepsilon(2 m+1)}$, and $E=n^{(2 m+1) d}$. Let $\mathcal{G}_{n, \varepsilon}$ be the set of bipartite random graphs such that all vertices in $V_{1}$ have degree at least $M_{n, \varepsilon}$. Then with probability tending to 1 as $n$ tends to infinity, $\Gamma\left(n^{m}, n^{m+1}, E\right)$ is in $\mathcal{G}_{n, \varepsilon}$.

Proof. Let $p=\frac{2}{3} n^{(2 m+1)(d-1)}$, so that that $\frac{3}{2} n^{2 m+1} p=E$. Then there exists a $d^{\prime} \in(1 / 2+\varepsilon, d)$ such that $p \geq n^{(2 m+1)\left(d^{\prime}-1\right)}$, so by Lemma $5.2, P\left(\Gamma\left(n^{m}, n^{m+1}, p\right) \in \mathcal{G}_{n, \varepsilon}\right) \rightarrow 1$ as $n \rightarrow \infty$.

Write $E_{p}$ for $\left|E\left(\Gamma\left(n^{m}, n^{m+1}, p\right)\right)\right|$. Since $n^{2 m+1} p=\omega(1)$, by Lemma 5.3,

$$
\delta_{n}:=P\left(E_{p} \notin\left[\frac{1}{2} n^{2 m+1} p, \frac{3}{2} n^{2 m+1} p\right]\right)
$$

satisfies $\delta_{n}=o(1)$. Hence, since the probability of being in $\mathcal{K}$ is only increased by adding edges,

$$
\begin{aligned}
P\left(\Gamma\left(n^{m}, n^{m+1}, p\right) \in \mathcal{G}_{n, \varepsilon}\right) & =\sum_{i=0}^{n^{2 m+1}} P\left(\Gamma\left(n^{m}, n^{m+1}, i\right) \in \mathcal{G}_{n, \varepsilon}\right) P\left(E_{p}=i\right) \\
& \leq \sum_{i=\frac{1}{2} n^{2 m+1} p} \sum^{\frac{3}{2} n^{2 m+1} p} P\left(\Gamma\left(n^{m}, n^{m+1}, i\right) \in \mathcal{G}_{n, \varepsilon}\right) P\left(E_{p}=i\right)+\delta_{n} \\
& \leq \sum_{i=\frac{1}{2} n^{2 m+1} p} \frac{3}{2} n^{2 m+1} p\left(\Gamma\left(n^{m}, n^{m+1}, E\right) \in \mathcal{G}_{n, \varepsilon}\right) P\left(E_{p}=i\right)+\delta_{n} \\
& \leq P\left(\Gamma\left(n^{m}, n^{m+1}, E\right) \in \mathcal{G}_{n, \varepsilon}\right)+\delta_{n} .
\end{aligned}
$$

Therefore, $P\left(\Gamma\left(n^{m}, n^{m+1}, E\right) \in \mathcal{G}_{n, \varepsilon}\right) \geq P\left(\Gamma\left(n^{m}, n^{m+1}, p\right) \in \mathcal{G}_{n, \varepsilon}\right)-\delta_{n} \rightarrow 1$ as $n \rightarrow \infty$.

Proof of Lemma 2.3. Let $0<\varepsilon<d-\frac{1}{2}$. Then by Lemma 5.4, with probability tending to 1 as as $n \rightarrow \infty$, each vertex in $V_{1}$ has degree at least $M_{n, \varepsilon}$.

Now, let $s_{1}, s_{2} \in V_{1}$, and consider the neighbourhoods $N\left(s_{1}\right), N\left(s_{2}\right) \subseteq V_{2}$. We calculate, using Lemma 4.9(ii),

$$
\begin{aligned}
P\left(N\left(s_{1}\right) \cap N\left(s_{2}\right)=\emptyset\right) & \leq \frac{\left(\begin{array}{c}
n^{m+1}-M_{n, \varepsilon} \\
M_{n, \varepsilon}
\end{array}\right)}{\left(\begin{array}{c}
n^{m+1} \\
M_{n, \varepsilon}
\end{array}\right)} \\
& \leq\left(1-\frac{M_{n, \varepsilon}}{n^{m+1}}\right)^{M_{n, \varepsilon}} \\
& =\left(1-n^{-m-1 / 2+\varepsilon(2 m+1)}\right)^{n^{1 / 2+\varepsilon(2 m+1)}} \\
& \leq\left(e^{-n^{-m-1 / 2+\varepsilon(2 m+1)}}\right)^{n^{1 / 2+\varepsilon(2 m+1)}} \\
& =e^{-n^{-m+2 \varepsilon(2 m+1)}} \\
& \leq 1-n^{-m+2 \varepsilon(2 m+1)}+\frac{1}{2} n^{-2 m+4 \varepsilon(2 m+1)} .
\end{aligned}
$$


Hence the probability that the two neighbourhoods intersect is at least

$$
n^{-m+2 \varepsilon(2 m+1)}-\frac{1}{2} n^{-2 m+4 \varepsilon(2 m+1)}=\frac{2 n^{m-2 \varepsilon(m+1)}-1}{2 n^{2 m-4 \varepsilon(2 m+1)}} .
$$

Choose $\gamma$ such that $0<\gamma<2 \varepsilon(2 m+1)$, and let $\mu=2 \varepsilon(2 m+1)-\gamma>0$. Then for sufficiently large $n$ the probability that the two neighbourhoods intersect is at least

$$
\frac{2 n^{m-2 \varepsilon(2 m+1)-\gamma}}{2 n^{2 m-4 \varepsilon(2 m+1)}}=\frac{1}{n^{m-\mu}} .
$$

Now define a graph $\Gamma^{\prime}$ with vertices $V_{1}$, and an edge between two vertices in $\Gamma^{\prime}$ if and only if the corresponding vertices in $\Gamma$ have a shared neighbour. Then $\Gamma^{\prime}$ is a random graph on $n^{m}$ vertices, with edge density at least $\frac{1}{n^{m-\mu}}$, and so by standard results due to Erdös and Rényi [2] the graph $\Gamma^{\prime}$ is connected. The result follows.

\section{References}

[1] S. Antoniuk, T. Łuczak and J. Światkowski, Random triangular groups at density 1/3, Compos. Math. 151 (2015), no.1, 167-78.

[2] P. Erdös and A. Rényi, On random graphs. I, Publ. Math. Debrecen 6 (1959), 290-297.

[3] M. Gromov, Asymptotic invariants of infinite groups, Geometric group theory, Cambridge University Press, 1993.

[4] M. Gromov, Random walk in random groups, Geom. Funct. Anal. 13 (2003), no. 1, 73-146.

[5] T. Johansson, The giant component of the random bipartite graph, Chalmers University of Technology, 2012, Master's Thesis.

[6] M. Kotowski and M. Kotowski, Random groups and property (T): Żuk's theorem revisited, J. Lond. Math. Soc., 88 (2013), 396-416.

[7] T. Odrzygóźdź, The square model for random groups, Colloq. Math. 142 (2016), 227-254.

[8] Y. Ollivier, Sharp phase transition theorems for hyperbolicity of random groups, Geom. Funct. Anal. 14 (2004), no. 3, 595-679.

[9] Y. Ollivier, A January 2005 invitation to random groups, Ensaios Matemáticos, vol. 10, Sociedade Brasileira de Matemática, Rio de Janeiro, 2005.

[10] Y. Ollivier, Some small cancellation properties of random groups, Internat. J. Algebra Comput. 17 (2007), no. 01, 37-51.

[11] Y. Ollivier and D. Wise, Cubulating random groups at density less than 1/6, Trans. Amer. Math. Soc. 363 (2011), no. 9, 4701-4733.

[12] I. Palasti, On the connectedness of bichromatic random graphs, Publ. Math. Inst. Hung. Acad. Sci 8 (1963), no. 341-440, 5.

[13] R. J. Serfling, Probability inequalities for the sum in sampling without replacement, Ann. Statist. 2 (1974), no. 1, 39-48. 
[14] A. Żuk, Property (T) and Kazhdan constants for discrete groups, Geom. Funct. Anal. 13 (2003), no. 3, 643-670.

Addresses:

DPMMS

Centre for Mathematical Sciences

Wilberforce Road

Cambridge

CB3 0WB

email: cja59@dpmms.cam.ac.uk

School of Mathematics and Statistics

University of St Andrews

St Andrews

Fife KY16 9SS

email: colva.roney-dougal@st-andrews.ac.uk 